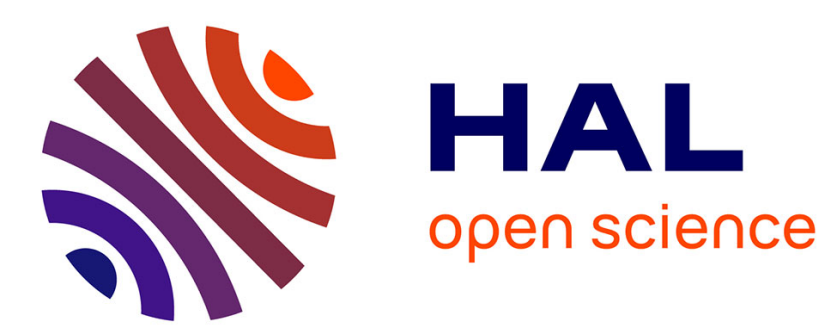

\title{
La métamémoire: analyse de sa mesure par questionnaire. Étude exploratoire
}

I. Fort

\section{To cite this version:}

I. Fort. La métamémoire: analyse de sa mesure par questionnaire. Étude exploratoire. Psychologie Française, 2005, 50 (2), pp.195 - 210. 10.1016/j.psfr.2004.08.001 . hal-01792141

\section{HAL Id: hal-01792141 https://hal-amu.archives-ouvertes.fr/hal-01792141}

Submitted on 16 May 2018

HAL is a multi-disciplinary open access archive for the deposit and dissemination of scientific research documents, whether they are published or not. The documents may come from teaching and research institutions in France or abroad, or from public or private research centers.
L'archive ouverte pluridisciplinaire HAL, est destinée au dépôt et à la diffusion de documents scientifiques de niveau recherche, publiés ou non, émanant des établissements d'enseignement et de recherche français ou étrangers, des laboratoires publics ou privés. 
La métamémoire : analyse de sa mesure par questionnaire. Etude exploratoire

Metamemory : analysis of its measure by questionnaire. Exploratory study

Isabelle FORT

Docteur en psychologie

Principaux thèmes de recherche : métamémoire, mémoire, vieillissement

GRAPCO- Laboratoire de Psychologie

Université Nancy 2 B.P. 3397

54015 Nancy Cedex

isafort5@aol.com

Tél. (personnel) : 0383364139 


\section{La métamémoire : analyse de sa mesure par questionnaire. Etude exploratoire}

Résumé : cette recherche vise à permettre l'élaboration d'un questionnaire de métamémoire. Une étude théorique du concept a permis de dégager quatre dimensions (les stéréotypes sur le vieillissement mnésique, les croyances du sujet sur ses aptitudes, ses connaissances relatives aux stratégies et l'utilisation de stratégies). Une analyse de contenu de questionnaires existant a mis en évidence une opérationnalisation incomplète de ces dimensions, et a permis l'identification de modalités spécifiques à celles-ci. Afin de vérifier la clarté de ces modalités, nous avons ensuite procédé à une comparaison inter juges sur un de ces questionnaires. Les pourcentages d'accord sont satisfaisants, à l'exception de deux modalités, mais les résultats montrent la nécessité de compléter cette étude par une étude similaire avec un autre questionnaire.

Mots clés : métamémoire, métacognition, analyse de contenu, méthode en facettes

\section{Metamemory : analysis of its measure by questionnaire. Exploratory study}

Abstract : this research aims at allowing an elaboration of a metamemory questionnaire. A study of metamemory and metacognition modelisations first allowed to identify four dimensions (the stereotypes about memory aging, the subject's beliefs about one's abilities, his knowledge about strategies and strategy use). A content analysis of available questionnaires evidenced an incomplete operationnalisation of these dimensions, and allowed us to identify some modalities for each of them. In order to check that modalities are clearly defined, 
we proceeded to a comparison between different subjects with one of these questionnaires. The agreement rates are satisfying, except for two modalities, but the results constrain us to complete this study with a similar one using another questionnaire.

Keywords : metamemory, metacognition, content analysis, facet method 


\section{Introduction}

La métamémoire est définie comme recouvrant d'une part les connaissances du sujet relatives au fonctionnement de la mémoire, d'autre part les processus de contrôle et d'autorégulation lui permettant d'orchestrer son activité mnésique et le contenu de sa mémoire (Boucheron, 1993). Une des raisons de l'intérêt croissant pour ce concept est la supposition selon laquelle il aiderait à expliquer le vieillissement des aptitudes mnésiques. Dans cette perspective, différentes hypothèses ont été suggérées (Devolder et Pressley, 1989 ; Hertzog, Dixon et Hultsch, 1990), pouvant se résumer de la manière suivante : les connaissances métamnésiques exerceraient chez les sujets âgés une influence négative sur les processus de contrôle et de régulation, et ainsi sur la performance. Or, dans la plupart des études empiriques sur ce thème, les auteurs se sont intéressés soit à la relation entre les processus de contrôle et de régulation et la performance, soit à l'influence directe des connaissances métamnésiques sur les performances (Abson et Rabbitt, 1988 ; Cavanaugh et Poon, 1989 ; Hertzog et al., 1990). En outre, les études ont donné lieu à des résultats divergents en fonction du questionnaire utilisé ou du domaine de métamémoire abordé. C'est le cas de l'étude de Zelinski, Gilewski, Anthony-Bergstone (1990) dans laquelle la dimension fréquence d'oubli du Memory Functioning Questionnaire est la plus fortement corrélée avec les performances. Cependant, ce résultat n'est pas répliqué dans l'étude de Taylor, Miller et Tinklenberg (1992). De même, l'étude de la relation entre l'utilisation de stratégies (dans la vie quotidienne) et les performances a donné lieu à des résultats divergents : cette relation n'est pas significative dans les études où le Memory 
Functioning Questionnaire est utilisé (Hertzog, Park, Morrell, Martin, 2000, Taylor et al 1992, Zelinski et al, 1990). A l'inverse, elle est significative dans certaines études utilisant le Metamemory In Adulthood, (Boucheron, 1993, Dixon, Hertzog et Hultsch, 1986). Ces résultats ont amené les auteurs à s’interroger sur la conceptualisation de la métamémoire et sur les qualités psychométriques des outils de mesure. Selon Dixon et Hertzog (1988), la métamémoire devrait être conçue comme regroupant plusieurs dimensions (se référant par exemple aux connaissances sur le fonctionnement de la mémoire en général et aux croyances du sujet sur ses aptitudes) et l'influence respective de ces dimensions sur les performances à des tâches de mémoire serait variable. Ces suggestions soulignent la nécessité d'une conceptualisation de la métamémoire, précisant les dimensions qui la constituent et leur pertinence dans l'explication des performances. Notre recherche s'inscrit dans cette perspective. Pour cela, nous allons tout d'abord nous intéresser à différentes théories de la métacognition et de la métamémoire afin d'identifier plusieurs dimensions. Une seconde étape visera à vérifier si ces dimensions se retrouvent dans la définition opérationnelle de la métamémoire, ceci grâce à une analyse de contenu de plusieurs questionnaires. Cette analyse de contenu permettra également de définir l'univers de contenu de ces dimensions théoriques à l'aide de la méthode en facettes. Nous nous assurerons ensuite de la clarté des facettes élaborées en demandant à quatre juges de classer les items d'un questionnaire existant en fonction de leurs modalités. Ce travail a un objectif essentiellement exploratoire, il permettra par la suite d'élaborer un outil de mesure de la métamémoire en adéquation avec les 
dimensions théoriques et d'étudier le rôle de chacune dans l'évolution des aptitudes mnésiques avec l’âge.

Nous nous sommes d'abord intéressée à différentes modélisations théoriques de la métacognition et de la métamémoire. Plusieurs critères de sélection ont été utilisés : nous avons retenu des théories qui mettent l'accent sur les connaissances métamnésiques, domaine qui a donné lieu à des résultats divergents, et qui incluent des hypothèses sur l'explication du fonctionnement mnésique (général ou spécifique aux sujets âgés). Ainsi avons-nous sélectionné le modèle de Flavell et Wellman (1977), celui de Schneider et Pressley (1989), celui de Hultsch, Hertzog, Dixon et Davidson (1988) et la définition proposée par Light (1996). Le tableau I présente les principales caractéristiques des théories retenues.

\section{Insérer Tableau I ici}

L'examen de ces modèles a fait apparaître quatre dimensions qui, selon les hypothèses posées, jouent un rôle dans l'explication des performances mnésiques des sujets âgés. La première dimension traite de l'opinion du sujet sur l'évolution de la mémoire avec l'âge : cette dimension est supposée influencer les croyances des sujets âgés relatives à leurs aptitudes mnésiques (Cavanaugh et Green, 1990 ; Light, 1996). La seconde dimension se réfère aux croyances du sujet concernant ses aptitudes de mémoire : elle est régulièrement évoquée comme facteur d'explication de l'évolution de ces aptitudes avec l'âge (Hultsch et al. 1988 ; Light, 1996). Cette dimension correspond à ce que Hertzog, Dixon et Hultsch 
(1990) désignent par efficacité personnelle liée à la mémoire et définie comme les croyances d'un sujet sur sa capacité à utiliser sa mémoire de manière efficiente dans différentes situations. La troisième dimension fait référence aux connaissances sur les stratégies. Dans le modèle de Schneider et Pressley (1989), chaque stratégie dont dispose un sujet peut être accompagnée de connaissances sur ses conditions d'utilisation et sur son utilité, ces connaissances étant inhérentes à une utilisation adéquate des stratégies. La dernière dimension concerne l'utilisation de stratégies : contrairement aux autres dimensions qui font partie des connaissances sur la mémoire, celle-ci fait partie des processus de contrôle et d'autorégulation mis en œuvre face à une épreuve de mémoire. Elle est considérée comme prépondérante dans la prédiction de la performance, par exemple dans le modèle de Schneider et Pressley (1989).

\section{Etude 1}

L'objectif de cette étude est non seulement de vérifier la présence des dimensions théoriques dans les questionnaires, mais également d'élaborer des modalités propres à chaque dimension.

\subsection{Méthode}

\subsubsection{Le corpus}

Afin de procéder à l'analyse de contenu des items de questionnaires, nous avons choisi de sélectionner non seulement des questionnaires de métamémoire, mais également des échelles de plainte mnésique (portant sur la fréquence d'oublis 
vécus par les sujets dans leur vie quotidienne), ainsi que des questionnaires ayant pour objectif d'évaluer la mémoire du sujet dans sa vie quotidienne. Le choix des questionnaires s'est porté sur des outils conçus pour un public adulte ou âgé afin d'assurer a priori une proximité maximale entre les dimensions théoriques identifiées ci-dessus et les outils de mesure. Le tableau II présente les questionnaires que nous avons recensés.

Insérer Tableau II ici.

\subsubsection{Analyse de contenu}

L'analyse de contenu a porté sur un corpus de 446 items provenant de 18 questionnaires. La méthode d'analyse de contenu utilisée est l'analyse thématique, au cours de laquelle, selon Jakobi et Wuillemain-Sales (1994, p. 220) “le codeur procède par repérage des différents thèmes ou spécifications contenus dans le texte puis par classement de ces thèmes dans des catégories plus générales ". L'unité de découpage est donc l'item. Mais comme nous allons nous intéresser en premier lieu à l'adéquation entre les dimensions théoriques et opérationnelles de la métamémoire, nous allons d'abord établir la liste des catégories plus générales, c'est-à-dire ce qu'évoquent globalement les items. Ensuite, nous dégagerons des thèmes spécifiques à ces catégories, autrement dit, nous identifierons différentes modalités à l'intérieur de chaque item, afin de délimiter l'univers de contenu des dimensions. 


\subsection{Résultats}

\subsubsection{Identification des catégories présentes dans les questionnaires}

L'identification des catégories abordées s'est faite intuitivement, au cours de l'étape de familiarisation avec le corpus. Le tableau III présente les catégories présentes dans les questionnaires sélectionnés.

$$
\text { Insérer tableau III ici }
$$

Le premier constat qui s'impose est la variabilité du nombre de catégories présentes dans les questionnaires : certains, comme le questionnaire de Zarit et al. (1981) évaluent seulement une catégorie, alors que d'autres, comme le Memory Questionnaire de Perlmutter (1978) permettent d'en identifier cinq. Ce constat montre que les auteurs ont des conceptions plus ou moins étendues de la métamémoire. Pour chaque catégorie recensée, nous avons calculé sa fréquence d'occurrence absolue et relative. Rappelons que l'examen des théories de la métamémoire a permis d'identifier quatre dimensions : l'opinion du sujet sur l'évolution de la mémoire avec l'âge, les croyances sur ses aptitudes, les connaissances à propos des stratégies et l'utilisation de stratégies. La première dimension théorique est présente dans seulement trois questionnaires. A l'inverse, la seconde dimension faisant référence à l'auto-évaluation des aptitudes est présente dans la moitié des questionnaires. Cependant, on peut considérer que les catégories se rapportant à la fréquence d'oubli, aux changements perçus dans les aptitudes et aux attentes de changement font également partie de cette dimension. Ainsi, la seconde dimension théorique 
apparaît-elle comme surreprésentée dans les questionnaires recensés (ces différents thèmes représentent $78 \%$ des catégories présentes). La troisième dimension théorique, ayant trait aux connaissances à propos des stratégies, n'est abordée dans aucun des questionnaires que nous avons recensés. Cette dimension n’a, à notre connaissance, pas été étudiée chez les sujets âgés, mais elle a fait l'objet de recherches chez de jeunes adultes (Huet, 1995) ou chez des enfants (Andreassen et Salatas Waters, 1989). La dernière dimension relative à l'utilisation de stratégies est présente dans cinq des questionnaires recensés. Il convient de souligner que les items des questionnaires évoquent les stratégies utilisées dans la vie quotidienne et que dans les modélisations théoriques, les auteurs font plutôt référence à la stratégie mise en œuvre par le sujet confronté à une épreuve de mémoire. Nous pouvons donc conclure de cette comparaison qu'il existe un décalage entre les définitions théorique et opérationnelle de la métamémoire. En effet, les connaissances sur les stratégies sont absentes des questionnaires, l'opinion du sujet sur l'évolution de la mémoire avec l'âge est sous-représentée, ses croyances sur ses aptitudes sont surreprésentées et l'utilisation des stratégies est présente dans la moitié des questionnaires. Ce constat montre la nécessité de procéder à une nouvelle opérationnalisation du concept de métamémoire.

\subsubsection{Définition de l'univers de contenu des dimensions}

Pour cela, nous avons utilisé la méthode en facettes qui permet, selon Dancer (1990, p367), “ de formuler une définition et de relier cette définition aux 
observations empiriques". Cette méthode permet de préciser ce que recouvre chaque dimension à l'aide de plusieurs facettes, comprenant chacune différentes modalités. Les facettes ont été obtenues grâce à l'analyse de contenu effectuée sur les items sélectionnés : dans chaque item, nous avons dégagé plusieurs thèmes qui constituent les facettes, notamment la polarité de l'item ou le type de mémoire auquel il se réfère. Ces facettes se déclinent suivant plusieurs modalités, par exemple la polarité de l'item comprend trois modalités suivant que l'item est rédigé en termes positifs, négatifs ou de stabilité. Chaque item doit pouvoir être classé en fonction de chaque facette et les modalités ont été construites de manière inductive, c'est-à-dire que c'est le contenu des items qui a guidé leur élaboration. Comme la méthode en facettes permet d'assurer le lien entre les définitions théorique et opérationnelle d'un concept, les facettes ainsi élaborées permettront de générer des items correspondant à une combinaison de leurs modalités.

Pour la dimension relative aux croyances du sujet sur ses aptitudes, nous avons identifié trois facettes faisant référence à la polarité de l'item, à la perspective temporelle évoquée et au type de mémoire abordé. Les modalités de ces facettes sont illustrées par des exemples d'items dans le tableau IV.

Insérer Tableau IV ici

Pour ce qui est de la facette 13. (type de mémoire évoqué), l'item était classé dans la modalité «mémoire »(131), lorsqu'il évoquait les aptitudes mnésiques de manière générale. Il était classé dans la modalité «mémoire à des tâches de 
laboratoire » (136), lorsqu'il il s'agissait de se souvenir de listes de mots ou de chiffres, comme par exemple dans les études expérimentales, alors qu'il était classé dans la modalité « mémoire dans la vie quotidienne »(137), lorsqu'il incluait le rappel de conversations, de contenus de livres, de films ou d'articles.

Pour la perception de l'évolution de la mémoire avec l'âge, nous retrouvons deux des trois facettes (ainsi que leurs modalités) identifiées pour la dimension précédente, à savoir : la polarité de l'item et le type de mémoire évoqué. La facette «perspective évoquée par l'item » n'a pas été conservée. Les deux facettes et leurs modalités sont présentées dans le tableau V.

Insérer Tableau V ici

Nous n'avons pas trouvé d'exemple d'items pour les modalités polarité positive et polarité stable, ni pour les modalités mémoire des visages, mémoire visuospatiale, mémoire prospective, mémoire lexicale, mémoire à des tâches de laboratoire et mémoire dans la vie quotidienne. Ce constat peut s'expliquer par le fait que les items relatifs à cette dimension évoquent les aptitudes mnésiques de manière générale, sans précision supplémentaire, et que ces modalités ont été élaborées à partir d'items traitant de la première dimension dans la perspective de l'élaboration d'un questionnaire. 
Concernant l'utilisation de stratégies, nous avons identifié deux facettes, se rapportant respectivement au type de stratégie et à sa finalité. Les modalités de ces facettes sont présentées dans le tableau VI.

\section{Insérer Tableau VI ici}

La modalité « faciliter l'encodage » (321) regroupe les stratégies pouvant être utilisées comme préparation pour une récupération future ; on peut citer comme exemples l'utilisation d'agenda, la planification des activités. La modalité « faciliter la récupération de l'information » inclut les stratégies visant à faciliter la récupération d'informations présentes en mémoire, par exemple : se souvenir de quelque chose de similaire à ce qu'on cherche ou reconstruire les événements de la journée.

Pour la dernière dimension théorique traitant des connaissances à propos des stratégies, nous ne disposons pas d'items permettant d'identifier des facettes pertinentes étant donné que cette dimension n'est pas évoquée dans les questionnaires utilisés pour l'analyse de contenu.

\section{Etude 2}

L'objectif de cette étude est de mettre à l'épreuve la clarté des dimensions théoriques et des modalités élaborées. Pour cela, nous avons présenté à quatre personnes non expertes dans le domaine de la métamémoire les dimensions théoriques, ainsi que les modalités des facettes. Leur tâche était d'indiquer à quelle dimension et à quelles modalités chaque item fait référence. Les résultats 
de cette étude permettront de déceler d'éventuelles ambiguïtés dans la définition de la métamémoire que nous avons élaborée.

\subsection{Méthodologie}

\subsubsection{Echantillon et procédure}

Le choix des codeurs s'est porté sur des personnes novices dans ce domaine car nous pensions que les modalités des facettes étaient compréhensibles par ce type de public. Ils sont au nombre de quatre (deux hommes et deux femmes) d'un âge moyen de 28 ans, titulaires d'un D.E.A. de psychologie. Cependant, étant donné la faiblesse de cet échantillon et sa non représentativité, les résultats de cette étude n'ont qu'un caractère exploratoire et ne pourront, en aucun cas, être généralisés à l'ensemble de la population.

Concrètement, les codeurs disposaient des dimensions théoriques et des modalités des facettes. Après avoir donné des explications sur ce que recouvrent, selon nous, ces différents éléments, il leur était demandé de classer par écrit les items sélectionnés en fonction de ces dimensions et des modalités des facettes. En cas de désaccord, nous leur avons demandé des explications sur ce qui a motivé leur classement. Nous avons ensuite calculé un pourcentage d'accord entre codeurs. Le choix de la statistique s'est porté sur le pourcentage d'accord car, vu le faible effectif de l'échantillon, il ne nous a pas paru nécessaire de calculer un coefficient d'accord, comme par exemple le kappa de Cohen.

\subsubsection{Questionnaire utilisé}


Dans cette étude, nous allons présenter les résultats obtenus sur les sous-échelles changement, capacité et stratégie de la version française du Metamemory In Adulthood (Boucheron, 1993). Nous avons choisi ce questionnaire car c'est le plus utilisé dans les études sur la relation entre métamémoire et mémoire, ainsi que dans les études sur l'évolution des connaissances métamnésiques avec le vieillissement. L'objectif qui a amené Dixon et Hultsch (1983) à élaborer ce questionnaire était de développer un instrument psychométrique à facteurs multiples. Dans cette perspective, ils ont élaboré huit sous-échelles : stratégie (utilisation de stratégies mnésiques), tâche (connaissance des processus et tâches de mémoire), capacité (connaissance de ses propres capacités), changement (perception de l'évolution de sa mémoire), activité (activités entretenant la mémoire), anxiété (mémoire et état d'anxiété), motivation (importance de la réussite à une tâche), locus (lieu de contrôle dans les aptitudes mnésiques). Ce questionnaire a fait l'objet de plusieurs études de ses qualités psychométriques, que ce soit dans sa version originale (Dixon et Hultsch, 1983) ou dans sa version française (Boucheron, 1993). Ces études ont permis de valider empiriquement la totalité des sous-échelles, à l'exception de celle nommée activité. Cependant, seules les sous-échelles capacité, changement et stratégie semblent correspondre aux dimensions théoriques que nous avons dégagées. C'est la raison pour laquelle les autres sous-échelles n'ont pas été administrées aux codeurs. Les items de ces sous-échelles sont présentés en annexe (les numéros indiqués correspondent à ceux de la version originale du questionnaire où les items des différentes souséchelles sont mélangés entre eux, c'est pourquoi ils ne se suivent pas). 


\subsection{Résultats}

\subsubsection{Identification des dimensions théoriques}

La première tâche des codeurs consistait à classer les items en fonction des quatre dimensions théoriques présentées dans l'introduction. Ils n'ont pas rencontré de difficultés, si bien qu'aucune erreur ou aucun désaccord interjuges n'a été constaté. Notons que de l'avis unanime des codeurs, l'item 28 fait davantage référence à l'opinion du sujet sur l'évolution de la mémoire avec l'âge, bien qu'il soit inclus dans une échelle traitant, selon les auteurs du MIA, de l'évolution perçue de ses aptitudes.

\subsubsection{Classement des items selon les modalités des catégories}

Après avoir classé l'item selon une dimension, les sujets devaient identifier les modalités des différentes facettes évoquées par ce dernier.

Pour les sous-échelles capacité et changement, les pourcentages d'accord sont relativement élevés. Plusieurs modalités de la facette relative au type de mémoire donnent lieu à des pourcentages d'accord de $100 \%$ pour la totalité des items concernés. Il s'agit des modalités mémoire (évaluée de manière globale : items 6, 9, 11, 13, 59, 62), mémoire prospective (item 29) et mémoire à des tâches de laboratoire (item 37). Cependant, ces deux dernières sont représentées seulement par un item, ce qui ne permet pas de conclure de manière fiable à la clarté de ces modalités. Remarquons par la même occasion que la modalité mémoire des visages n'est pas représentée dans ce questionnaire. Les modalités mémoire dans 
la vie quotidienne (items 21, 32, 33, 36, 41, 67, 70, 73, 74) et mémoire lexicale (items 2, 40, 61, 66) donnent lieu également à des pourcentages d'accord de $100 \%$, sauf pour les items 38 et 23 . L'item 38 a mis en évidence un désaccord à propos du rappel de dates. C'est pour la modalité mémoire visuo-spatiale (items $27,28,44)$ que l'on observe le plus de désaccords. En effet, des confusions ont été observées entre cette modalité et la modalité mémoire dans la vie quotidienne. Pour certains codeurs, en effet, le fait de retrouver un objet implique de retrouver l'endroit où il est (d'où le classement des items dans la modalité mémoire visuospatiale), alors que pour d'autres, ce rappel implique le rappel des actions effectuées avant de le poser (d'où le classement des items dans la modalité mémoire dans la vie quotidienne).

En ce qui concerne la perspective et la polarité, peu de divergences entre codeurs sont apparues. Les items demandant une auto-évaluation des aptitudes mnésiques par rapport à une perspective actuelle (items 2, 33, 36, 41, 44, 61, 66, 67, 70, 73, 74) ou passée (items $6,9,13,21,23,27,29,32,38,40,59,62$ ) donnent lieu à des pourcentages d'accord de $100 \%$. Il en est de même pour les items rédigés en termes positifs (items $2,32,33,36,44,59,61,66,67,70,73,74$ ) ou en termes négatifs (items 9, 11, 21, 23, 27, 28, 29, 37, 38, 40, 41, 62). Les deux autres modalités de ces catégories sont très peu représentées : il s'agit des items rédigés en termes de stabilité (items 6 et 13) et des items demandant une auto-évaluation par rapport à une perspective future (item 37). Bien que les pourcentages d'accord concernant ces modalités varient entre 75 et $100 \%$, l'examen de leur objectivité demanderait confirmation. 
Concernant la sous-échelle stratégie, la distinction entre stratégies internes (items $4,12,39,42,46,48,54,58$ ) et stratégies externes (items 19, 22, 26, 65, 68, 76) semble bien établie et donne lieu à des pourcentages d'accord optimaux pour tous les items. Les modalités traitant de la finalité de la stratégie sont moins clairement identifiées : nous obtenons des pourcentages d'accord de 100\% seulement pour une partie des items (items 4, 39, 42, pour les stratégies visant à faciliter la récupération, items $46,48,54,58$, pour les stratégies visant à faciliter l'encodage). D'autres items fournissent des pourcentages d'accord plus modérés (de 25 à 75\%), par exemple ceux évoquant l'utilisation d'un tableau d'affichage ou le fait de laisser un objet en vue. En effet, ces comportements sont considérés par certains codeurs comme favorisant la récupération de l'information au moment voulu et par d'autres comme favorisant l'encodage car le sujet y a recours au moment où il prend conscience de la nécessité de retenir l'information. La distinction établie par Flavell et Wellman (1977) entre stratégies pouvant être utilisées pour faciliter une récupération future et stratégies utilisées pour favoriser une récupération présente paraît plus adaptée pour définir ces modalités.

\section{Discussion}

Dans cette étude, nous avons proposé une définition de la métamémoire tenant compte des dimensions invoquées comme facteurs d'explication du vieillissement mnésique et de son opérationnalisation actuelle. A partir de plusieurs théories de la métamémoire et de la métacognition, nous avons identifié quatre dimensions d'ordre général. Ces dimensions ont été comparées aux catégories présentes dans 
plusieurs questionnaires. Une analyse de contenu thématique a mis en évidence des résultats différents suivant la dimension considérée : 1'opinion du sujet sur l'évolution de la mémoire avec l'âge est sous-représentée, ses croyances sur ses aptitudes sont surreprésentées, les connaissances à propos des stratégies n'apparaissent pas dans les questionnaires recensés et l'utilisation de stratégies est également sous-représentée. En outre, cette dernière dimension ne semble pas définie de manière similaire dans les modèles théoriques et dans l'opérationnalisation de la métamémoire par questionnaire. L'analyse de contenu a ensuite permis de délimiter l'univers de contenu des dimensions théoriques en faisant apparaître des modalités spécifiques à chacune d'elles. Afin de vérifier la clarté de la définition obtenue, plusieurs dimensions théoriques et leurs modalités ont fait l'objet d'une comparaison inter juges. Les pourcentages d'accord relativement élevés indiquent que les dimensions validées (les croyances du sujet sur ses aptitudes mnésiques et l'utilisation des stratégies) sont clairement distinguées par les codeurs. De même, plusieurs modalités apparaissent comme définies de manière claire, il s'agit des modalités mémoire, mémoire lexicale, mémoire dans la vie quotidienne, des perspectives passée et actuelle et des polarités positive et négative. La clarté d'autres modalités, quant à elle, devra être confirmée à cause du faible nombre d'items s'y référant dans cette étude (il s'agit de la mémoire prospective, à des tâches de laboratoire, de la perspective future et des items rédigés en termes de stabilité). Une étude analogue s'avère donc nécessaire avec d'autres questionnaires pour évaluer la clarté des modalités peu présentes dans le MIA De même, la dimension relative à l'opinion du sujet sur 
l'évolution de la mémoire au cours du vieillissement devra être validée par une étude similaire. Les pourcentages d'accord sont plus faibles pour les stratégies utilisées pour favoriser l'encodage et pour la mémoire visuo-spatiale. Ces modalités devront donc être redéfinies afin d'éviter toute ambiguïté. Par ailleurs, une des dimensions théoriques (faisant référence aux connaissances à propos des stratégies) n'est pas du tout opérationnalisée dans les questionnaires que nous avons recensés. Cette dimension a été étudiée chez de jeunes adultes (Huet, 1995) et chez des enfants (Andreassen et Salatas Waters, 1989) sous la forme de questions ouvertes. Huet demandait à des apprentis de classer différentes stratégies par ordre d'efficience et Andreassen et Salatas Waters demandaient à leurs sujets d'indiquer quelle stratégie ils conseilleraient à un autre enfant. Il conviendra donc 1) de s'inspirer de ces études pour opérationnaliser cette dimension et 2) soumettre cette opérationnalisation à une étude de validation. De telles études complémentaires s'avèrent indispensables pour permettre la mise au point d'un questionnaire qui, élaboré à partir des quatre dimensions dégagées et des modalités établies, devrait donner lieu à une meilleure adéquation entre les définitions théoriques et opérationnelles de la métamémoire. Le recours à un tel questionnaire devrait permettre de valider empiriquement des hypothèses théoriques sur la relation entre métamémoire et mémoire, comme par exemple les hypothèses qui ont été posées (voir tableau I) sur l'explication du vieillissement mnésique par la métamémoire et sur l'influence que certaines dimensions peuvent exercer sur d'autres. Ainsi, il paraît judicieux de s'intéresser au rôle des stéréotypes sur les croyances concernant ses aptitudes (Cavanaugh et Green, 
1990), au rôle de ces croyances sur l'utilisation de stratégies (Hertzog et al., 1990)

et à l'influence de l'utilisation des stratégies sur les performances (Light, 1996).

De même, comme le suggère Dixon (2000), il paraît souhaitable de savoir comment les différentes dimensions de la métamémoire interagissent pour déterminer les performances mnésiques. Cependant, la métamémoire comprend également les processus de contrôle et d'autorégulation, considérés en outre comme une variable intermédiaire entre les connaissances métamnésiques et la performance de mémoire (Hultsch et al., 1988 ; Light, 1996). Ces processus ne doivent donc pas être négligés dans la mise à l'épreuve de ces hypothèses, ils peuvent être opérationnalisés notamment par le temps d'étude ou le sentiment de savoir.

\section{Références}

Abson, V., Rabbitt, P., 1988. What do self-rating questionnaires tell us about changes in competences in old age. In: Gruneberg, M.M. Morris, P.E. Sykes, R.N. (Eds.), Practical aspects of memory: Volume 2. Current research and issues. Wiley and Sons, Chichester, pp. 186-191.

Andreassen, C., Salatas Waters, H., 1989. Organization during study:

Relationships between metamemory, strategy use and performance. Journal of Educational Psychology, 81, 190-195.

Bennett-Levy, J., Powell, G.E., 1981. The Subjective Memory Questionnaire (SMQ): An investigation into the self-reporting of "real-life" memory skills. British Journal of Social and Clinical Psychology, 19, 177-88. 
Berry, J.M., West, R.L., Dennehey, D.M., 1989. Reliability and validity of the memory self-efficacy questionnaire. Developmental Psychology, 25, 701-713. Boucheron, C., 1993. Mémoire, métamémoire et vieillissement. Thèse de doctorat. Université Nancy 2.

Broadbent, D.E., Cooper, P.F., Fitzgerald, P., Parkes, K.R., 1982. The Cognitive Failures Questionnaire (CFQ) and its correlates. British Journal of Social and Clinical Psychology, 21, 1-16.

Cavanaugh, J.C., 1986-87. Age differences in adults' self reports of memory ability: it depends on how and what you ask. International Journal of Aging and Human Development, 24, 271-277.

Cavanaugh, J.C., Green, E.E., 1990. I believe, therefore I can: self-efficacy believes in memory aging. In: Lovelace, E.A. (Ed.), Aging and cognition: mental process, self awareness and interventions. Elsevier Science Publishers, pp.189230.

Cavanaugh, J.C., Poon, L.W., 1989. Metamemorial predictors of memory performance in young and older adults. Psychology and Aging, 4, 365-368. Dancer, S., 1990. Introduction to facet theory and its applications. Applied Psychology: An international review, 39(4), 365-377.

De Rotrou, J., Adjémian, A., Ouanhnon, P., Kurz, H., Moulin, F., Forette, F., 1996. Le Pac Eureka. In: Michel, B.F. De Rotrou, J. Verdureau, F. (Eds), La stimulation cognitive, activation, rééducation, stimulations cérébrales et mesures objectives. Solal, Marseille, pp. 163-175. 
Devolder, P.A., Pressley, M., 1989. Metamemory across the adult lifespan. Canadian Psychology, 30, 578-587.

Dixon, R.A., 2000. The concept of metamemory: Cognitive, developmental and clinical issues. In: Berrios, G.E. Hodges, J.R. (Eds.), Memory disorders in psychiatric practice. Cambridge University Press, New-York, pp. 47-57. Dixon, R.A., Hertzog, C., 1988. A functional approach to metamemory development in adulthood. In: Weinert, F.E. Perlmutter, M. (Eds.), Memory developement: Universal changes and individual differences. Erlbaum, Hillsdale, pp.293-330.

Dixon, R.A., Hertzog, C., Hultsch, D.F., 1986. The multiple relationships among Metamemory In Adulthood (MIA) scales and cognitive abilities in adulthood. Human Learning, 5, 165-177.

Dixon, R.A., Hultsch, D.F., 1983. Structure and development of metamemory in adulthood. Journal of Gerontology, 38, 682-688.

Flavell, J.H., Wellman, H.M., 1977. Metamemory. In: Kail R.V. Hagen J.W. (Eds.), Perspectives of the development of memory and cognition. Erlbaum, Hillsdale, pp. 3-33.

Gilewski, M.J., Zelinski, E.M., 1988. Memory Functioning Questionnaire. Psychopharmacological Bulletin, 24, 665-669.

Herrmann, D.J., Neisser, U., 1978. An inventory of everyday memory experiences. In: Gruneberg, M.M. Morris, P. Sykes, R.N. (Eds.), Practical aspects of memory. Academic Press, London, pp. 35-51. 
Hertzog, C., Dixon, R.A., Hultsch, D.F., 1990. Relationships between metamemory, memory predictions and memory task performances in adults. Psychology and Aging, 5, 215-227.

Hertzog, C., Park, D., Morrell, R., Martin, M., 2000. Ask and ye shall receive: Behavioural specificity in the accuracy of subjective memory complaints. Applied Cognitive Psychology, 14, 257-275.

Huet, N., 1995. Connaissances métamnésiques et autorégulation de l'activité cognitive sous l'effet de contraintes mnémoniques. Thèse de doctorat de Psychologie. Université Toulouse II.

Hultsch, D.F., Hetzog, C., Dixon, R.A., Davidson, H., 1988. Memory selfknowledge and self-efficacy in the aged. In: Howe, M.L. Brainerd, C.J. (Eds), Cognitive Development in Adulthood: Progress in cognitive development research. Springer Verlag, New York, pp. 65-92.

Jakobi, J.M., Wuillemin-Sales, E., 1994. Méthodes utilisant le langage. In: Ghiglione, R. Richard, J.F. (Eds.), Cours de psychologie, Tome 4: Mesures et analyses. Dunod, Paris, pp. 167-269.

Johansson, B., 1985. Memory and memory measurement in old age: Memory structure, context and metamemory. Jönköping, Sweden, Institute of gerontology. Larrabee, G.J., Levin, H.S., 1986. Memory self-ratings and objective test performance in a normal elderly sample. Journal of Clinical Experimental Neuropsychology, 8, 275-284. 
Larrabee, G.J., West, R.B., Crook, T., 1991. The association of memory complaint with computer simulated everyday memory performance. Journal of Clinical and Experimental Neuropsychology, 13, 466-478.

Light, L.L., 1996. Memory and aging. In: Ligon Bjork, E., Bjork, R.A. (Eds), Memory. Handbook of perception and cognition, San Diego, Academic Press, pp. 444-490.

Niederehe, G. Yoder, C., 1989. Metamemory perceptions in depressions of young and older adults. The journal of nervous and mental disease, 77, 4-14.

Perlmutter, M., 1978. What is memory aging the aging of ? Developmental Psychology, 14, 330-345.

Poitrenaud, J., Israel, L., Barrère, H., Le Roch, K., 1997. Version française de l'échelle de difficultés cognitives de Mac-Nair et Kahn. In: Michel, B.F.

Dérouesné, C. Gely-Nargeot, M.C. (Eds.), De la plainte mnésique à la maladie d'Alzheimer. Solal, Marseille, pp. 159-168.

Schneider, W., Pressley, M., 1989. Memory development between 2 and 20. Springer Verlag, New York.

Sunderland, A., Harris, J.E., Baddeley, A.D., 1983. Do laboratory tests predict everyday memory? A neuropsychological study. Journal of Verbal Learning and Verbal Behavior, 22, 341-357.

Taylor, J.L., Miller, T.P., Tinklenberg, J.R., 1992. Correlates of memory decline: a 4-year longitudinal study of older adults with memory complaints. Psychology and Aging, 7, 185-193. 
Van Der Linden, M., Wyns, C., Coyette, F., Von Frenkel, R., Seron, X., 1989. Le QAM, questionnaire d'auto-évaluation de la mémoire. Editest, Bruxelles.

Williams, S.A., Denney, N.W., Schadler, M., 1983. Elderly adults' perception of their own cognitive development during the adult years. International Journal of Aging and Human Development, 16, 147-158.

Zarit, S.H., Cole, K.D., Guider, R.L., 1981. Memory training strategies and subjective complaints of memory in the aged. The Gerontologist, 21, 158-164. Zelinski, E.M., Gilewski, M.J., Anthony-Bergstone, C.R., 1990. Memory Functioning Questionnaire: concurrent validity with memory failures. Psychology and Aging, 5, 388-399.

\section{Annexes}

Sous-échelle capacité du MIA

2. Je me souviens bien des noms.

33. Je me souviens bien de l'ordre dans lequel ont lieu les événements.

36. Je me souviens bien des conversations auxquelles j'ai participé.

41. J'oublie souvent qui était avec moi lors des événements auxquels j'ai participé.

44. Je me souviens bien des endroits où je suis allé(e).

61. Je me souviens bien des titres de livres, de films ou de pièces de théâtre.

66. Je me souviens bien des titres de morceaux de musique.

67. Quand j'ai lu un livre, je n'ai pas de mal à me souvenir d'informations contenues dans celui-ci. 
70. Je me souviens bien du contenu des articles de journaux ou de celui d'émissions de radio ou de télévision.

73. Je me souviens bien de la trame des histoires ou des romans.

74. En général, je me souviens exactement où j'ai lu ou entendu quelque chose.

Sous-échelle changement du MIA

6. Actuellement, je me souviens aussi bien qu'autrefois.

9. Maintenant, je me souviens moins bien qu'autrefois.

11. Plus je vieillis et plus il est difficile de me souvenir clairement.

13. Ma mémoire est tout aussi bonne qu'elle a été.

21. Je me souviens beaucoup moins bien maintenant du contenu d'un article de journal ou d'une émission de radio ou de télé qu'il y a 10 ans.

23. Je me souviens beaucoup moins bien des titres de livres, de films ou de pièces de théâtre qu'il y a 10 ans.

27. J'égare plus souvent des objets maintenant que lorsque j'étais jeune.

28. Au fur et à mesure que l'on vieillit, on a tendance à oublier plus fréquemment l'endroit où l'on a rangé les objets.

29. Par rapport à une dizaine d'années, j'oublie maintenant beaucoup plus souvent mes rendez-vous.

32. Durant ces 10 dernières années, ma mémoire des événements importants s'est améliorée.

37. Ma mémoire des numéros de téléphone déclinera au fur et à mesure que je vieillirai.

38. Depuis 10 ans, ma mémoire des dates a énormément décliné. 
40. Depuis une dizaine d'années, ma mémoire des noms a énormément décliné.

59. Ma mémoire s'est beaucoup améliorée durant ces 10 dernières années.

62. Ma mémoire a énormément décliné au cours de ces 10 dernières années.

Sous-échelle stratégie du MIA

4. Lorsque vous cherchez quelque chose que vous avez récemment égaré, essayez-vous de retracer mentalement votre parcours pour le localiser?

12. En début de journée, pensez-vous aux activités que vous devez accomplir afin de vous souvenir de tout ce que vous avez à faire?

19. Placez-vous des notes rappelant ce que vous devez faire à un endroit bien en vue, comme un tableau d'affichage par exemple?

22. Mettez-vous régulièrement les objets au même endroit afin de les retrouver plus facilement quand vous en avez besoin?

26. Quand vous avez à emporter quelque chose avec vous, le laissez-vous dans un endroit bien en évidence (par exemple poser une valise devant la porte)?

39. Quand vous avez du mal à vous souvenir de quelque chose, essayez-vous de vous rappeler autre chose de similaire afin de vous aider?

42. Reconstruisez-vous consciencieusement les événements de la journée pour vous aider à retrouver quelque chose?

46. Essayez-vous de relier une chose que vous voulez retenir à une autre dans l'espoir d'améliorer vos chances de vous en souvenir plus tard?

48. Essayez-vous de vous concentrer très fort sur quelque chose que vous voulez retenir?

54. Construisez-vous des images mentales pour vous aider à retenir? 
58. Vous répétez-vous mentalement quelque chose que vous essayez de retenir?

65. Ecrivez-vous des notes "aide mémoire"?

68. Ecrivez-vous vos rendez-vous sur un calendrier pour vous aider à vous en souvenir?

76. Ecrivez-vous vos listes d'achats? 
Tableau I : principales caractéristiques des modèles de la métamémoire retenus pour identifier les dimensions théoriques

\begin{tabular}{||l|l|l||}
\hline Auteurs & Dimensions constitutives & Hypothèses concernant les effets de la \\
métamémoire sur la performance
\end{tabular}




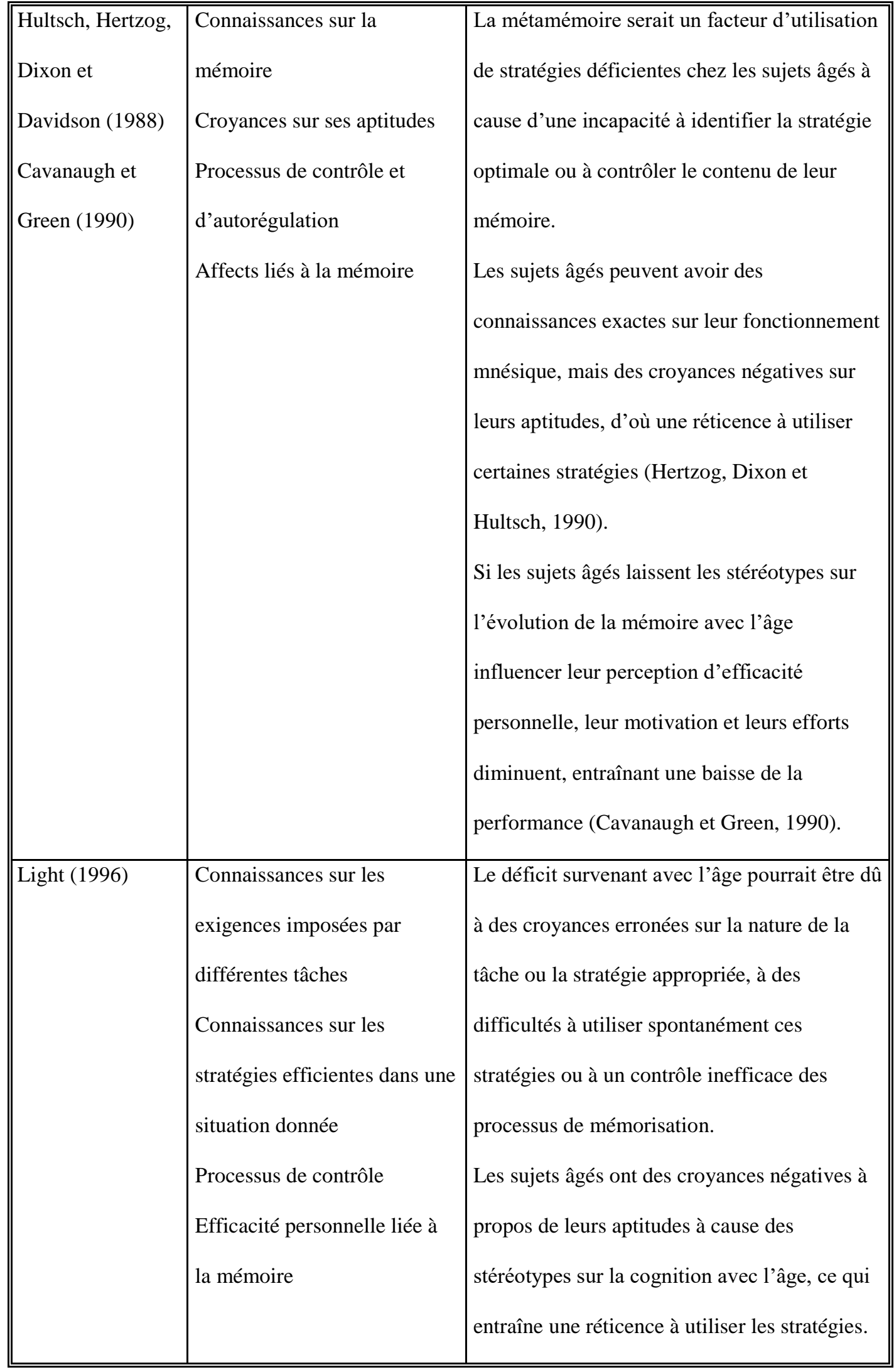


Tableau II : questionnaires utilisés pour l'analyse de contenu

\begin{tabular}{|c|c|}
\hline Questionnaire & $\begin{array}{l}\text { Nombre } \\
\text { d'items }\end{array}$ \\
\hline Questionnaire de Zarit, Cole, Guider (1981) & 8 \\
\hline Subjective Memory Questionnaire (SMQ) de Bennett-Levy et Powell (1981) & 40 \\
\hline $\begin{array}{l}\text { Cognitive Failures Questionnaire (CFQ) de Broadbent, Cooper, Fitzgerald, Parkes } \\
\text { (1982) }\end{array}$ & 19 \\
\hline Questionnaire de Sunderland, Harris, Baddeley (1983) & 35 \\
\hline $\begin{array}{l}\text { Exemples d'items de l'Inventory of Memory Experiences (IME) de Herrmann et } \\
\text { Neisser (1978) }\end{array}$ & 60 \\
\hline $\begin{array}{l}\text { Traduction de l'échelle de difficultés cognitives de Mc Nair et Kahn (Poitrenaud, } \\
\text { Israel, Barrère, Le Roch, 1997) }\end{array}$ & 25 \\
\hline MEMO 50 (De Rotrou, Adjemian, Ouanhnon, Kurz, Moulin, Forette, 1996) & 34 \\
\hline Questionnaire de Williams, Denney, Schadler (1983) & 5 \\
\hline Exemples d'items du questionnaire de Larrabee et Levin (1986) & 5 \\
\hline Memory Questionnaire (MQ) de Perlmutter (1978) & 34 \\
\hline Exemples d'items du questionnaire de Niederehe et Yoder (1989) & 5 \\
\hline Exemples d'items du questionnaire de Cavanaugh (1986-87) & 15 \\
\hline $\begin{array}{l}\text { Questionnaire d'Auto-évaluation de la Mémoire (QAM) de Van Der Linden, Coyette } \\
\text { et Von Frenkel (1989) }\end{array}$ & 48 \\
\hline Memory Functioning Questionnaire (MFQ) de Gilewski et Zelinski (1988) & 45 \\
\hline $\begin{array}{l}\text { Metamemory in Adulthood (MIA) de Dixon et Hultsch (1983), traduit par Boucheron } \\
\text { (1993) }\end{array}$ & 40 \\
\hline Questionnaire de Johansson (1985) & 8 \\
\hline Exemples du MAC S (Larrabee, West et Crook, 1991) & 10 \\
\hline Memory Self-Efficacy Questionnaire (MSEQ) de Berry, West et Dennehey (1989) & 10 \\
\hline Total & 446 \\
\hline
\end{tabular}


Tableau III : catégories mises en évidence par l'analyse de contenu thématique

\begin{tabular}{|c|c|}
\hline Questionnaire & Catégories présentes \\
\hline Questionnaire de Zarit et al. (1981) & Auto-évaluation des aptitudes \\
\hline $\begin{array}{l}\text { Subjective Memory Questionnaire (SMQ) de Bennett-Levy } \\
\text { et Powell (1981) }\end{array}$ & $\begin{array}{l}\text { Auto-évaluation des aptitudes } \\
\text { Fréquence d'oubli }\end{array}$ \\
\hline $\begin{array}{l}\text { Cognitive Failures Questionnaire (CFQ) de Broadbent et al. } \\
\text { (1982) }\end{array}$ & Fréquence d'oubli \\
\hline Questionnaire de Sunderland et al. (1983) & Fréquence d'oubli \\
\hline $\begin{array}{l}\text { Exemples d'items de l'Inventory of Memory Experiences } \\
\text { (IME) de Herrmann et Neisser (1978) }\end{array}$ & $\begin{array}{l}\text { Fréquence d'oubli } \\
\text { Auto-évaluation des aptitudes }\end{array}$ \\
\hline $\begin{array}{l}\text { Traduction de l'échelle de difficultés cognitives de Mc Nair } \\
\text { et Kahn (Poitrenaud et al., 1997) }\end{array}$ & Fréquence d'oubli \\
\hline MEMO 50 (De Rotrou et al., 1996) & Fréquence d'oubli \\
\hline Questionnaire de Williams et al. (1983) & $\begin{array}{l}\text { Changements perçus } \\
\text { Evolution de la mémoire avec l'âge }\end{array}$ \\
\hline $\begin{array}{l}\text { Exemples d'items du questionnaire de Larrabee et Levin } \\
\text { (1986) }\end{array}$ & Changements perçus \\
\hline Memory Questionnaire (MQ) de Perlmutter (1978) & $\begin{array}{l}\text { Utilisation de stratégies } \\
\text { Fréquence d'oubli } \\
\text { Auto-évaluation des aptitudes } \\
\text { Changements perçus } \\
\text { Attentes de changement }\end{array}$ \\
\hline $\begin{array}{l}\text { Exemples d'items du questionnaire de Niederehe et Yoder } \\
\text { (1989) }\end{array}$ & $\begin{array}{l}\text { Fréquence d'oubli } \\
\text { Attentes de changement } \\
\text { Evolution de la mémoire avec l'âge } \\
\text { Utilisation de stratégies }\end{array}$ \\
\hline
\end{tabular}




\begin{tabular}{|c|c|}
\hline $\begin{array}{l}\text { Exemples d'items du questionnaire de Cavanaugh (1986- } \\
\text { 87) }\end{array}$ & $\begin{array}{l}\text { Changements perçus } \\
\text { Auto-évaluation des aptitudes } \\
\text { Utilisation de stratégies } \\
\text { Fréquence d'oubli }\end{array}$ \\
\hline $\begin{array}{l}\text { Questionnaire d'Auto-évaluation de la Mémoire (QAM) de } \\
\text { Van Der Linden et al. (1989) }\end{array}$ & Fréquence d'oubli \\
\hline $\begin{array}{l}\text { Memory Functioning Questionnaire (MFQ) de Gilewski et } \\
\text { Zelinski (1988) }\end{array}$ & $\begin{array}{l}\text { Fréquence d'oubli } \\
\text { Changements perçus } \\
\text { Utilisation de stratégies }\end{array}$ \\
\hline $\begin{array}{l}\text { Metamemory in Adulthood (MIA) de Dixon et Hultsch } \\
\text { (1983), traduit par Boucheron (1993) }\end{array}$ & $\begin{array}{l}\text { Auto-évaluation des aptitudes } \\
\text { Utilisation de stratégies } \\
\text { Changements perçus }\end{array}$ \\
\hline Questionnaire de Johansson (1985) & $\begin{array}{l}\text { Auto-évaluation des aptitudes } \\
\text { Changements perçus } \\
\text { Evolution de la mémoire avec l'âge }\end{array}$ \\
\hline Exemples du MAC S (Larrabee et al., 1991) & $\begin{array}{l}\text { Auto-évaluation des aptitudes } \\
\text { Fréquence d'oubli }\end{array}$ \\
\hline $\begin{array}{l}\text { Memory Self-Efficacy Questionnaire (MSEQ) de Berry et } \\
\text { al. (1989) }\end{array}$ & Auto-évaluation des aptitudes \\
\hline
\end{tabular}


Tableau IV : modalités mises en évidence pour les croyances sur ses aptitudes mnésiques

\begin{tabular}{|c|c|c|}
\hline$\overline{\text { Facettes }}$ & Modalités & Exemple d'item \\
\hline \multirow{3}{*}{ 11. polarité de } & 111. positif & Je me souviens bien des noms (MIA, Boucheron, 1993). \\
\hline & 112. négatif & $\begin{array}{l}\text { Il m'arrive d'oublier des rendez-vous (MEMO 50, De } \\
\text { Rotrou et al., 1996). }\end{array}$ \\
\hline & 113. stable & $\begin{array}{l}\text { Ma mémoire est tout aussi bonne qu'elle a été (MIA, } \\
\text { Boucheron, 1993). }\end{array}$ \\
\hline \multirow{3}{*}{$\begin{array}{l}\text { 12. perspective } \\
\text { évoquée par l'item }\end{array}$} & 121. passée & $\begin{array}{l}\text { Est-ce que votre mémoire a changé par rapport à quand } \\
\text { vous étiez plus jeune? (questionnaire de Zarit et al., 1981). }\end{array}$ \\
\hline & 122. future & $\begin{array}{l}\text { Ma mémoire des numéros de téléphone déclinera au fur et } \\
\text { à mesure que je vieillirai (MIA, Boucheron, 1993). }\end{array}$ \\
\hline & 123. actuelle & $\begin{array}{l}\text { Globalement, pensez-vous que vous avez une bonne ou une } \\
\text { mauvaise mémoire? (questionnaire de Johansson, 1985) }\end{array}$ \\
\hline \multirow{5}{*}{$\begin{array}{l}\text { 13. type de mémoire } \\
\text { évoqué par l'item }\end{array}$} & 131. mémoire & $\begin{array}{l}\text { Avez-vous des problèmes de mémoire ? (questionnaire de } \\
\text { Niederehe et Yoder, 1989) }\end{array}$ \\
\hline & $\begin{array}{l}\text { 132. mémoire } \\
\text { des visages }\end{array}$ & $\begin{array}{l}\text { Je n'arrive pas à reconnaître des gens que je connais. } \\
\text { (traduction de l'échelle de difficultés cognitives de Mc } \\
\text { Nair et Kahn par Poitrenaud et al., 1997) }\end{array}$ \\
\hline & $\begin{array}{l}\text { 133. mémoire } \\
\text { visuo-spatiale }\end{array}$ & $\begin{array}{l}\text { Supposez que vous retourniez à un endroit où vous n'êtes } \\
\text { allé qu'une fois auparavant, auriez-vous à demander } \\
\text { l'itinéraire ? (IME, Herrmann et Neisser, } 1978 \text { ) }\end{array}$ \\
\hline & $\begin{array}{l}\text { 134. mémoire } \\
\text { prospective }\end{array}$ & $\begin{array}{l}\text { Oubliez-vous souvent des rendez-vous? (MQ, Perlmutter, } \\
1978)\end{array}$ \\
\hline & $\begin{array}{l}\text { 135. mémoire } \\
\text { lexicale }\end{array}$ & $\begin{array}{l}\text { Trouvez-vous que vous oubliez le nom des gens ? (CFQ, } \\
\text { Broadbent et al., 1982) }\end{array}$ \\
\hline
\end{tabular}




\begin{tabular}{||l|l|l||}
\hline \hline & $\begin{array}{l}\text { 136. mémoire à } \\
\text { des tâches de } \\
\text { laboratoire }\end{array}$ & $\begin{array}{l}\text { J'oublie le numéro de mon compte bancaire ou de ma carte } \\
\text { de crédit, ou mon numéro de sécurité sociale (MEMO 50, } \\
\text { De Rotrou et al., 1996). }\end{array}$ \\
\cline { 2 - 4 } & $\begin{array}{l}\text { 137. mémoire } \\
\text { dans la vie }\end{array}$ & Avez-vous des difficultés à lire parce que vous oubliez au \\
quotidienne & fur et à mesure ce que vous venez de lire, ce qui vous \\
\hline
\end{tabular}


Tableau V : modalités mises en évidence pour l'opinion du sujet sur l'évolution de la mémoire avec l'âge

\begin{tabular}{|c|c|c|}
\hline Facettes & Modalités & Exemple d'item \\
\hline \multirow{3}{*}{$\begin{array}{l}\text { 21. polarité de } \\
\text { l'item }\end{array}$} & 211. positif & \\
\hline & 212. négatif & $\begin{array}{l}\text { Au fur et à mesure que l'on vieillit, on a tendance à } \\
\text { oublier plus fréquemment l'endroit où l'on a rangé } \\
\text { les objets (MIA, Boucheron, 1993). }\end{array}$ \\
\hline & 213. stable & \\
\hline \multirow{7}{*}{$\begin{array}{l}\text { 22. type de } \\
\text { mémoire } \\
\text { évoqué par } \\
\text { l'item }\end{array}$} & 221. mémoire & $\begin{array}{l}\text { Vous m'avez parlé de ce que vous pensez qu'il est } \\
\text { arrivé à votre mémoire quand vous avez vieilli. Que } \\
\text { pensez-vous qu'il arrive à la mémoire de la plupart } \\
\text { des gens quand ils vieillissent ? (questionnaire de } \\
\text { Williams et al., 1983) }\end{array}$ \\
\hline & 222. mémoire des visages & \\
\hline & $\begin{array}{l}\text { 223. mémoire visuo- } \\
\text { spatiale }\end{array}$ & $\begin{array}{l}\text { Au fur et à mesure que l'on vieillit, on a tendance à } \\
\text { oublier plus fréquemment l'endroit où l'on a rangé } \\
\text { les objets (MIA, Boucheron, 1993). }\end{array}$ \\
\hline & 224. mémoire prospective & \\
\hline & 225. mémoire lexicale & \\
\hline & $\begin{array}{l}\text { 226. mémoire à des tâches } \\
\text { de laboratoire }\end{array}$ & \\
\hline & $\begin{array}{l}\text { 227. mémoire dans la vie } \\
\text { quotidienne }\end{array}$ & \\
\hline
\end{tabular}


Tableau VI : modalités mises en évidence pour l'utilisation de stratégies

\begin{tabular}{||l|l|l||}
\hline Facettes & Modalités & Exemple d'item \\
\hline 31. Type de & 311. interne & Répétez-vous souvent mentalement quelque chose que vous \\
& essayez de vous rappeler ? (MQ, Perlmutter, 1978) \\
\cline { 2 - 4 } & 312. externe & Ecrivez-vous souvent vos rendez-vous sur un calendrier pour \\
\hline \multirow{3}{*}{ 32. Finalité de stratégie } & $\begin{array}{l}321 . \text { faciliter } \\
\text { l'encodage }\end{array}$ & $\begin{array}{l}\text { Construisez-vous des images mentales pour vous aider à retenir } \\
\text { ? (MIA, Boucheron, 1993). }\end{array}$ \\
\cline { 2 - 4 } & 322. faciliter la & Lorsque vous cherchez quelque chose que vous avez récemment \\
& récupération & égaré, essayez-vous de retracer mentalement votre parcours pour \\
& le localiser ? (MIA, Boucheron, 1993). \\
\hline \hline
\end{tabular}

